\title{
ELECTROCHEMICALLY-FUNCTIONALIZED AND VERTICALLY CONDUCTIVE ADHESIVE TAPES FOR WEARABLE SWEAT BIOMARKER MONITORING Yichao Zhao ${ }^{1}$, Hannaneh Hojaiji ${ }^{2}$, and Sam Emaminejad ${ }^{2}$ \\ ${ }^{1}$ Department of Materials Science and Engineering, University of California, Los Angeles, California, USA \\ ${ }^{2}$ Department of Electrical Engineering, University of California, Los Angeles, California, USA
}

\section{ABSTRACT}

A vertically conductive and electrochemically functionalized adhesive sensor is developed, which can be simply taped onto/detached from a wearable circuit board's sensor-designated contact pads. These sensors are used in human subject testing experiments to monitor the sweat metabolites' concentrations.

\section{INTRODUCTION}

The exponential growth in the Internet of Things (IoT) devices and wearable sensing technologies have created an unprecedented opportunity to enable personalized medicine, through real-time biomonitoring of individuals and enabling actionable feedback. Currently, commercialized IoT devices and wearable sensors are only capable of tracking physical activities and vital signs and fail to access molecular-level biomarker information to provide insight into the body's dynamic chemistry. Sweat-based wearable biomonitoring has emerged as one of the most promising candidates to merge this gap. Sweat is a rich source of biomarkers that can be retrieved unobtrusively [1]. Previously reported sweat analysis platforms have successfully demonstrated the in-situ measurement of sweat analytes in wearable formats. However, the lack of suitable sensor fabrication/integration schemes continue to prevent us from incorporating these sensors into commercialized wearable technologies to scale them for population-level adoption. Specifically, the presented platforms comprise of physicallydecoupled sensor arrays and readout circuit board modules and rely on 2D electrical connections (on the same plane as the sensing interface) and cables to relay the transduced signal. Therefore, inherently, they are spatially inefficient and their integration into existing wearable technologies is non-trivial. To overcome these bottlenecks, here, we devise a novel sensor fabrication/integration methodology, which allows for seamless and compact integration of disposable electrochemical sensors with permanent readout electronics.

\section{RESULTS AND DISCUSSION}

As conceptualized in Fig. 1, in our approach, we construct our electrochemical sensing layer on a vertically-conductive adhesive substrate that can be simply taped onto/detached from the electrical contact pads of a wearable electronic reader. As a proof of concept, we applied our methodology to create enzymatic glucose and lactate

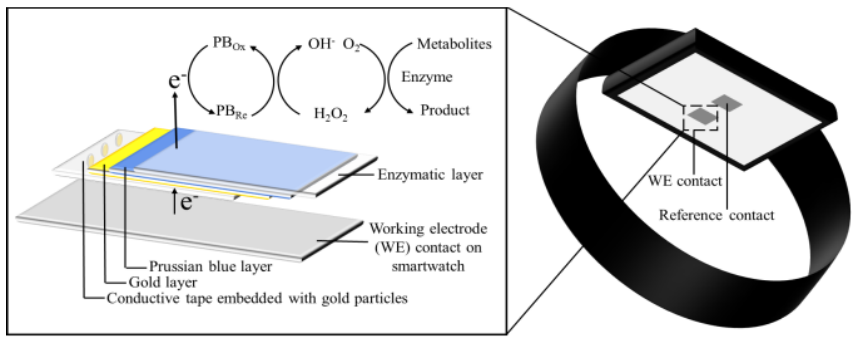

Figure 1: Schematic diagram for a representative enzymatic sensor, where the disposable working electrode (WE) can be simply taped onto the corresponding metal contact of the backside of a smartwatch (as an electronic reader). The placement of the reference electrode follows the same procedure. sensors and validated their functionalities by performing human sweat sample analysis. To construct our sensing layer, we first evaporate gold on a z-axis electrically-conductive adhesive tape (which exploits gold particles, embedded in its structure, for electron transfer in vertical direction). Then, the gold-coated surface is functionalized with glucose/lactate oxidase enzymes entrapped in chitosan films. These sensing interfaces effectively output electrical current correlating to the concentration of the target analytes. Because of the sensor structure's unique z-direction electron transfer property, and stable adhesion to metal contacts or common printed circuit board substrates (including gold and copper), the electrochemically-functionalized tape can be vertically integrated into electronic platforms (e.g. smartwatch).

Figure 2 illustrates the calibration curves for the glucose and lactate sensors, demonstrating the corresponding sensors' highly linear responses $\left(R^{2}=0.99\right)$ within the physiologically relevant ranges of concentrations.
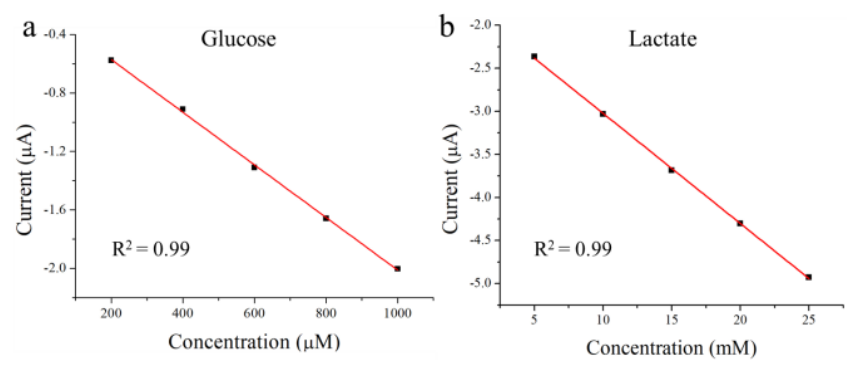

Figure 2: The calibration curves of glucose (a) and lactate (b) sensors (characterized in phosphate-buffered saline) demonstrating the high degree of linearity of the sensors' responses.
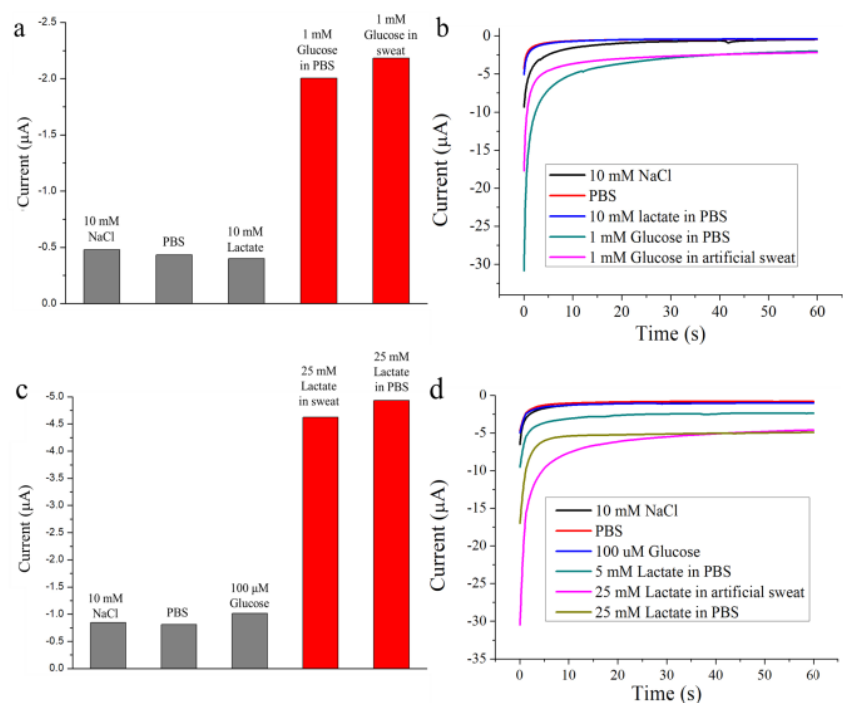

Figure 3: Interference study of glucose sensor response: steady state (a) and corresponding amperometric response (b). The equivalent interference study figures for lactate sensor are shown in (c) and (d). Hilton Head Island, South Carolina, June 3-7, 2018 
We validated our sensors' selectivity, by studying the effect of non-target compounds (present in sweat) on our sensors' responses. As can be seen in Fig. 3, the sensors' output current levels due to interfering molecules are negligible as compared to those generated in response to target analytes.

To validate our glucose sensor functionality, we collected iontophoretically-stimulated sweat samples from three subjects during 12 -h fasting and $0.5 \mathrm{~h}$ after glucose intake ( $30 \mathrm{~g}$ glucose). As shown in Fig. 4, the sweat glucose level is clearly increased in all three subjects. Therefore, it is a promising method to achieve non-invasive blood glucose monitoring.
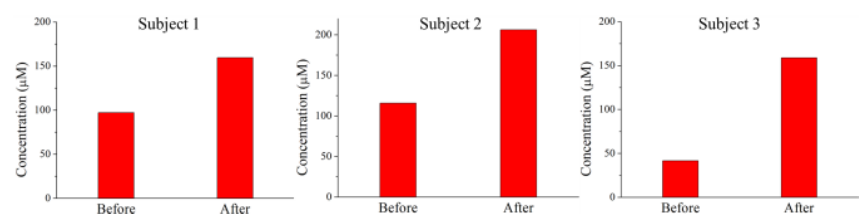

Figure 4: Sweat glucose levels of three subjects during 12-h fasting state and 30 minutes after $30 \mathrm{~g}$ of glucose intake.

Additionally, we integrated our lactate sensor into a smartwatch to perform real-time sweat analysis during a graded-load cycling exercise (Fig. 5). In this study, the exercise intensity was increased at two steps (700 and $900 \mathrm{~s}$ after beginning the exercise). The sweat secretion initiated at about $800 \mathrm{~s}$ after beginning the exercise. The wirelessly transmitted sweat lactate information demonstrated that the readily stabilized sweat lactate concentration elevated in response to the second increase in the exercise intensity level (as expected).

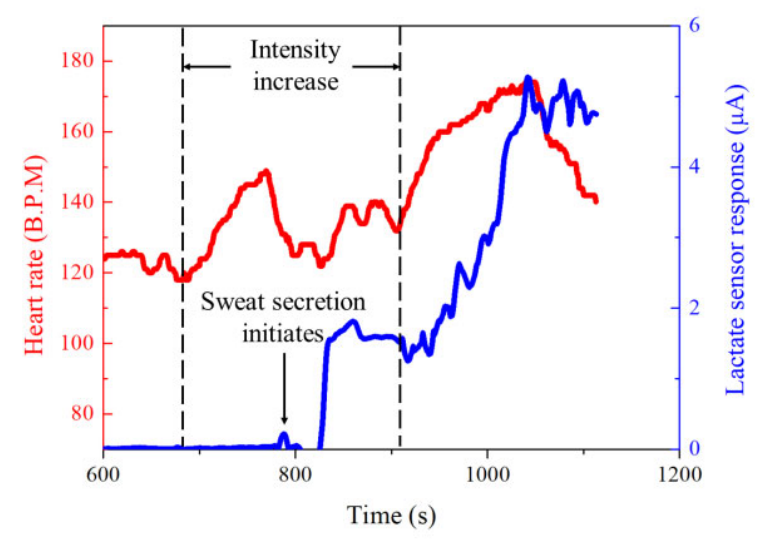

Figure 5: On-body sweat lactate measurement during physical exercise (stationary cycling with three different intensities). The measured readings are low-pass filtered at $0.1 \mathrm{~Hz}$ in digital domain. The upper curve indicates the heart rate profile, measured by a commercial heart rate sensor (left axis) and the lower curve shows the sweat lactate concentration profile (right axis). The exercise intensity was increased at two steps (700 s and $900 \mathrm{~s}$ after beginning the exercise), which immediately followed by increase in the measured heart rate. The sweat secretion initiated at about $800 \mathrm{~s}$ after beginning the exercise and the sweat lactate level was elevated in response to the second increase in power intensity.

Our scalable sensor fabrication and seamless integration methodology paves the way for incorporation of sweat sensors in commercialized wearable technologies for general population health monitoring.

\section{REFERENCES}

[1] S. Emaminejad, W. Gao, H. Y. Y. Nyein, S. Challa, R. W. Davis, A. Javey, "Flexible Systems for Wearable Physiological Monitoring Applications", Solid State Sensors, Actuators, and Microsystems Conference, Hilton Head Isl., 06 (2016) pp. 108-109.

\section{CONTACT}

*S. Emaminejad, emaminejad@ucla.edu 